\title{
Fully Automatic Lung Segmentation and Rib Suppression Methods to Improve Nodule Detection in Chest Radiographs
}

\author{
Elaheh Soleymanpour ${ }^{1}$, Hamid Reza Pourreza ${ }^{2}$, Emad ansaripour ${ }^{3}$, Mehri Sadooghi Yazdi ${ }^{4}$
}

\begin{abstract}
:
Computer-aided Diagnosis (CAD) systems can assist radiologists in several diagnostic tasks. Lung segmentation is one of the mandatory steps for initial detection of lung cancer in Posterior-Anterior chest radiographs. On the other hand, many CAD schemes in projection chest radiography may benefit from the suppression of the bony structures that overlay the lung fields, e.g. ribs. The original images are enhanced by an adaptive contrast equalization and non-linear filtering. Then an initial estimation of lung area is obtained based on morphological operations and then it is improved by growing this region to find the accurate final contour, then for rib suppression, we use oriented spatial Gabor filter. The proposed method was tested on a publicly available database of 247 chest radiographs. Results show that this method outperformed greatly with accuracy of $96.25 \%$ for lung segmentation, also we will show improving the conspicuity of lung nodules by rib suppression with local nodule contrast measures. Because there is no additional radiation exposure or specialized equipment required, it could also be applied to bedside portable chest x-rays. In addition to simplicity of these fully automatic methods, lung segmentation and rib suppression algorithms are performed accurately with low computation time and robustness to noise because of the suitable enhancement procedure.
\end{abstract}

\section{KEY WORDS:}

Adaptive enhancement, chest radiograph, lung segmentation, morphological operation, rib suppression, spatial Gabor.

\section{1- INTRODUCTION}

At the present time, chest radiography is the most common type of screening procedure for the initial detection of every type of abnormal pulmonary condition, due to its ability of revealing some unsuspected pathologic alterations; it's noninvasively characteristics, radiation dose and economic considerations. Nevertheless, this is the most difficult radiograph to produce technically and to interpret diagnostically, as discussed in [1].

The first and mandatory step of an automatic system aimed at any type of computerized analysis on chest radiographs, is the lung field segmentation. Typically, several lung segmentation methods based on classical techniques have been reviewed in [2].

On the other hand, there are several reasons why automated analysis of chest radiographs can benefit from suppression of bony structures. A study showed that most lung cancer lesions that are missed on frontal chest radiographs are located behind the ribs and that the inspection of a soft tissue image can improve detection performance by humans [3]. Automated lung cancer detection schemes also suffer from false positives caused by superposition of bony structures [4]. Including analysis of simulated soft-tissue images may improve performance of lung nodule detection systems.

1- E.soleymanpour is with the Machine Vision Research Laboratory, Computer Engineering Department, Ferdowsi University of Mashhad, Iran (E-mail: e.soleymanpour@yahoo.com).

2- H.R.Pourreza is with the Machine Vision Research Laboratory, Computer Engineering Department, Ferdowsi University of Mashhad, Iran (E-mail: hpourreza@ieee.org).

3- E.Ansaripour is with the radiology department, Mashhad University of Medical Sciences, Mashhad, Iran.(emad ansaripour @ yahoo.com) 
In Section 5.B we investigate if the conspicuity of lung nodules increases when applying the bone suppression technique. Other abnormalities such as subtle patterns of interstitial disease may also be detected, classified and quantified more accurately if the bony anatomy is adequately suppressed. Finally, automatic segmentation of bony structures may benefit from enhancing them [4].

We proposed a fully automatic method, which enhance the original image as a preprocessing step and then segment it by a sequence of morphological operations. With no need of training or human interaction, the proposed method has lower time complexity than the other techniques. After that, we efficiently suppress rib cage in obtained lung area with oriented spatial Gabor filter. Our purpose was to develop an image processing technique for suppressing the contrast of ribs in chest radiographs to improve nodule detection.

The method has been developed and tested on 247 radiographs (154 of patients with lung nodules) in the standard JSRT database [5], which is the only known standard database publicly available. The obtained results are compared with lung masks that prepared by Dr. Bram van Ginneken [6].

The reminder of this paper is organized as follows: In section 2, we introduce related works with lung segmentation and rib suppression methods. In section 3, we will describe spatial Gabor filter as Preliminaries. In section 4, the proposed method is described in detail. Experimental results are discussed in section 5, and we investigate if the conspicuity of lung nodules increases when applying the rib suppression technique. Finally, the paper concludes in section 5 .

\section{2- RELATED WORK:}

Segmentation of lung fields in PA (PosteriorAnterior) chest radiographs has received considerable attention in the literature. The two main approaches are rule-based reasoning and pixel classification with neural networks. Rule-based systems include algorithms consisting of a series of steps, each containing specific processing and, usually, certain adjustable parameters [2]. Several rule-based schemes have been proposed by $\mathrm{Li}$ et al. [7], Armato et al. [8], $\mathrm{Xu}$ and Doi [9], Duryea and Boone [10], Pietka [11], and Brown et al. [12]. Lung segmentation by pixel classification using neural networks has been investigated by McNitt- Gray et al. [13] and Tsujii et al. [14] .Vittitoe et al. [15] developed a pixel classifier for the identification of lung regions using Markov random field modeling. An iterative pixel-based classification method related to Markov random fields was presented by Loog and van Ginneken [6]. Van Ginneken and TerHaarRomeny [16] proposed a hybrid method that combines a rule-based scheme with a pixel classifier. ASM (Active Shape Modeling) has been used for lung field segmentation by van Ginneken et al. [17], [18]. An ASM consists of a shape model controlling, a set of landmark points, together with a statistical model of the grey-levels expected around each landmark. In [19], an ASM based on deformed method is applied to segment the lung region. Three supervised segmentation methods for lung segmentation are compared in [20]: active shape models, active appearance models and a multi-resolution pixel classification method that employs a multi-scale filter bank of Gaussian derivatives and a k-nearest-neighbors classifier.

All of the above-mentioned methods for lung segmentation have some drawbacks. For instance, rulebased methods are parametric algorithms, which require a priori knowledge obtained from an expert. Lung segmentation based on pixel classification methods require training phase with prepared lung masks. The last one, ASM, is a semiautomatic method, which an expert has to determine a set of landmark points on images. Therefore, time complexity of these approaches is high because of human interaction or including training phase.

An attempt was made in [21] where the rib cage was suppressed by means of Massive Training Artificial Neural Network (MTANN). Separation of bone and tissue components in chest radiographs can be obtained by dual energy imaging in which in addition to the normal exposure a second image is acquired at a lower energy level. Because the X-ray attenuation coefficients of bone (containing calcium) and soft-tissue have different energy dependence, a properly calibrated system can separate these two components from these two acquisitions [22]. A drawback of the system is the need of a dual-energy bone image in which the ribs are separated from the soft-tissue at the initial training phase. The technique was found to be sensitive to the noise levels, due to the subtraction process. A reduction of the contrast of the ribs in chest radiographs is reported, but it is not mentioned how contrast is measured.

Loog et al in [4] presented a novel framework for 
image filtering based on regression. They applied regression on a pixel level. A new, substantially different, image is estimated from an input image by computing a number of filtered input images (feature images) and mapping these to the desired output for every pixel in the image. The total scheme consists of preprocessing, feature computation, feature extraction by a novel dimensionality reduction scheme designed specifically for regression, regression by k-nearest neighbor averaging, and (optionally) iterative application of the algorithm. The framework is applied to estimate the bone and soft-tissue components from standard frontal chest radiographs. As training material, radiographs with known soft-tissue and bone components, obtained by dual energy imaging, are used. The results show that good correlation with the true softtissue images can be obtained and that the scheme can be applied to images from a different source with good results. We show that bone structures are effectively enhanced and suppressed and that in most soft-tissue images local contrast between pulmonary nodules and their surroundings increases; making them relatively more pronounced.

\section{3- ORIENTED SPATIAL GABOR FILTER:}

Spatial frequencies and their orientations are important characteristics of textures in images. Fig 1 shows examples of spatial textures with characteristic frequency and orientations. The frequency characteristics of images can be analyzed using spectral decomposition methods like Fourier analysis. A key problem with Fourier analysis is that spectral features from different parts of the image are mixed together. Many image analysis applications e.g. object recognition, tracking, etc., require spatially localized features. Gabor filters are a popular tool for this task of extracting spatially localized spectral features [23].

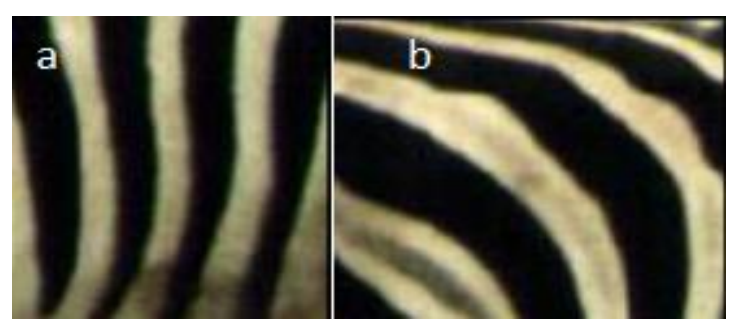

Figure 1: Example of spatial frequencies in images: (a)

Vertical stripes the frequencies would have horizontal orientation, and (b) Curved stripes[23]
A spatial Gabor filter [24] is obtained by modulating a sinusoid with a Gaussian. For the case of onedimensional (1D) signals, a 1D sinusoid is modulated with a Gaussian. This filter will therefore respond to some frequency, but only in a localized part of the signal. For 2D signals such as images, consider the sinusoid. By combining this with a Gaussian, we obtain a Gabor filter. Let $g(x, y, \theta, \varphi)$ be the function defining a spatial Gabor filter centered at the origin $(\mathrm{x}, \mathrm{y})$ with $\theta$ as the spatial frequency and $\varphi$ as the orientation. We can view spatial Gabor filters as:

$$
\begin{aligned}
\mathrm{g}(\mathrm{x}, \mathrm{y}, \theta, \varphi)= & \exp \left(-\left(\left(\mathrm{x}^{2}+\mathrm{y}^{2}\right) /\left(\sigma^{2}\right)\right) *\right. \\
& \exp (2 \pi \theta \mathrm{i}(\mathrm{x} \cos \varphi+\mathrm{y} \sin \varphi))
\end{aligned}
$$

It has been shown that $\sigma$, the standard deviation of the Gaussian kernel depends upon the spatial frequency to measured, i.e. $\theta$. In our case, $\sigma=0.65 \theta$.

The response of a spatial Gabor filter to an image is obtained by a 2D convolution operation. Let $\mathrm{I}(\mathrm{x}, \mathrm{y})$ denote the image and $\mathrm{G}(\mathrm{x}, \mathrm{y}, \theta, \varphi)$ denote the response of a Gabor filter with frequency $\theta$ and orientation $\varphi$ to an image at point $(\mathrm{x}, \mathrm{y})$ on the image plane. $\mathrm{G}$ (.) is obtained as:

$$
\mathrm{G}(\mathrm{x}, \mathrm{y}, \theta, \varphi)=\iint \mathrm{I}(\mathrm{p}, \mathrm{q}) \mathrm{g}(\mathrm{x}-\mathrm{p}, \mathrm{y}-\mathrm{q}, \theta, \varphi)
$$

\section{4- PROPOSED METHOD:}

Our proposed method involves preprocessing step as image enhancement process, lung segmentation and finally, rib suppression:

- $\quad$ Lung image enhancement: Original image has low contrast and further image processing techniques may end up with undesired results, so an initial step of contrast enhancement seems to be necessary. The result of this step is an enhanced image in which the lung area is clearly distinct from background. So if we do not enhance the image, the accurate segmentation cannot be obtained.

- Accurate lung segmentation: segmentation is done in two steps. At first, an initial segmented image is estimated, and then the accurate segmentation is performed with morphological operations and thresholding. 
After examining different structuring element types, disk shaped ones had the best results. Since the width of ribs almost is equal to 10 pixels in this resolution $(512 * 512)$, structuring elements with radius of 5 resulted most accurate as expected. Therefore, in our approach, we defined a disk shaped structuring element with 5 pixels radius which was used for all morphological operations.

- Rib suppression: we use spatial Gabor filter based on vertical orientation to approximate ribs' location. Then we apply subtraction method followed by smoothing with adaptive Gaussian filter to remove noise and artifact.

Fig2 shows the main steps of our proposed method in lung segmentation and rib suppression phases.

Each of these major steps has different parts (as it can be seen from Fig2) that will be described next in detail.

\section{A. Lung enhancement:}

Lung image enhancement is performed by three steps:

- Global contrast enhancement: since the overall illumination of images in the database is varying in a wide range, we used adaptive histogram equalization to normalize their histograms. This step normalizes contrast across different images (Fig3 (b)).

- Local contrast enhancement: To normalize the contrast within each image, we perform local contrast enhancement (LCE) identical to one presented in [25]. This operation is given by

$Y(x, y)=\frac{I(x, y)-\mu(x, y)}{\sigma(x, y)}$

Where $\mathrm{I}(\mathrm{x}, \mathrm{y})$ is the input image, $\mathrm{Y}(\mathrm{x}, \mathrm{y})$ is the LCE image, $\mu(x, y)$ is a local mean estimate, and $\sigma(x, y)$

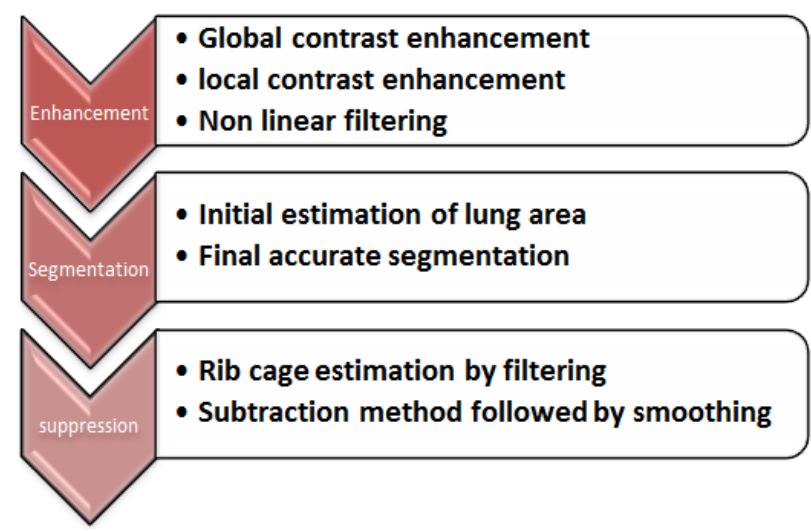

Figure2. Schematic diagram of the proposed method is a local standard deviation estimate. The local mean is computed by

$$
\mu(x, y)=I(x, y) * h(x, y)
$$

Where $\mathrm{h}(\mathrm{m}, \mathrm{n})$ is a Gaussian low-pass filter with an impulse response function having a standard deviation of 16 (corresponding to a one standard deviation diameter span of $22.4 \mathrm{~mm}$ according to average size of nodules in database in resolution $512 * 512$ ). The local standard deviation is computed by (fig3 (c ))

$$
\sigma(x, y)=\sqrt{I^{2}(x, y)^{*} h(x, y)-\mu^{2}(x, y)}
$$

- Nonlinear filtering: A morphological open operation for signal enhancement and a ring-shaped median filter for signal suppression are applied separately and then obtained results are subtracted to make an enhanced version of image by

NonLinearFilter $=\operatorname{morphOpen}(\mathrm{img})-\operatorname{median} 2 \mathrm{D}(\mathrm{img})$

Fig3 shows the original and enhanced images for JPCLN100; that is an example image of JSRT database.

\section{B. Accurate segmentation}

Initial segmented image is estimated by following operations: Binary image is obtained by Otsu method [26]. To avoid effect of borders on image processing we have to remove borders. An opening morphological operation is used to clear borders of image to isolate lung area from borders of the image After that, connected components of this binary image are extracted, and then two largest components with most area are selected as initial estimation of lungs.

Stomach gases, which usually can be seen in the left diaphragm, are wrongly segmented with a part of the left lung field, so an opening morphological operation with introduced structuring element is applied. After that, two regions with the biggest major axis are remaining. In order to reduce the area to be processed, we used the convex hull of the obtained areas. Moreover most the methods presented in the literature are indeed detected the most visible parts of the left and right lung (visible lung areas), excluding those hidden behind the diaphragm, the heart and the spinal column (hidden lung areas), where tumors could be found [1]. 


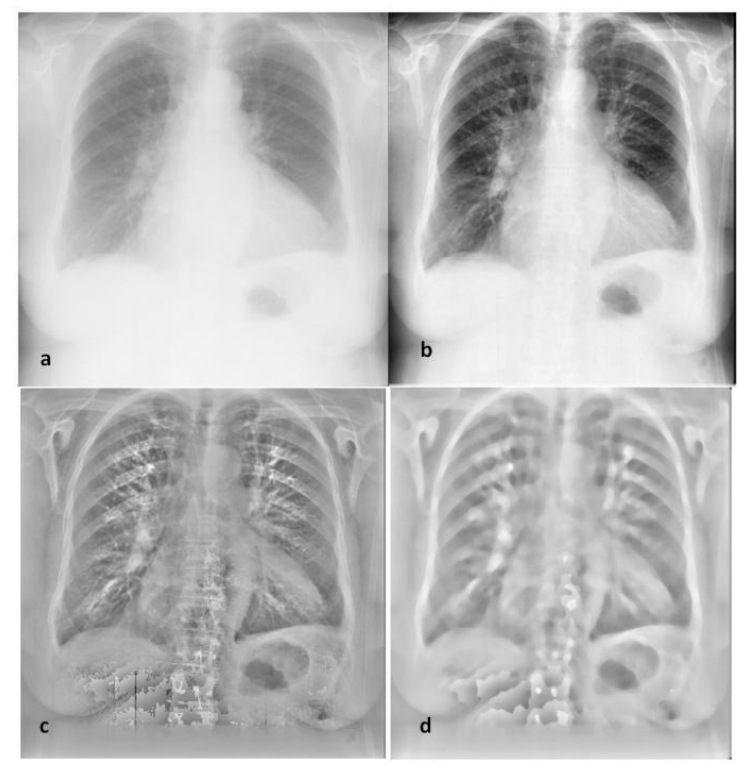

Figure3. (a) Original image. (b) Result of global contrast enhancement. (c) Result of local contrast enhancement. (d) Result of nonlinear filtering

Then the convex hull is used as a mask to apply Otsu method [22] on enhanced image. At the end, a morphological dilation is applied to reduce False Negative (FN) area with introduced structuring element is applied. The initial estimated and the final segmented images are shown in Fig 4.

\section{Rib suppression}

A major challenge in current $\mathrm{CAD}$ schemes for nodule detection on chest radiographs is the detection of nodules overlapping with ribs, rib crossings, and clavicles, because a majority of false positives are caused by these structures. This results in lowering the sensitivity as well as the specificity of a CAD scheme. Because nodules overlapping with ribs and clavicles were reported to be difficult for radiologists to observe, detection of such nodules is important for CAD scheme.

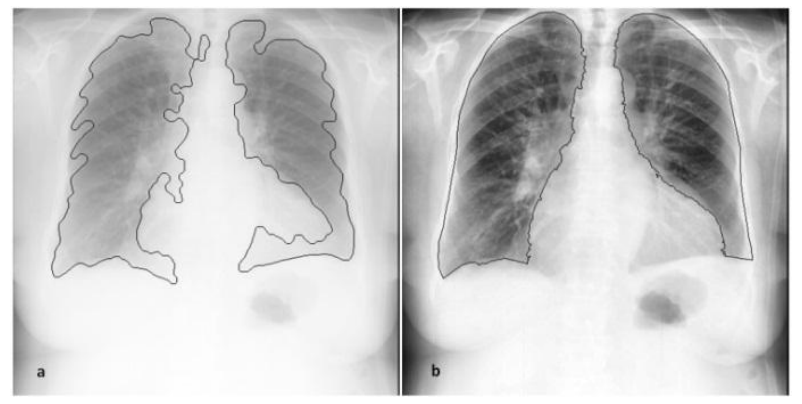

Figure 4. (a) Initial and (b) Final accurate segmentation
Therefore, the suppression of ribs and clavicles in chest radiographs would be potentially useful for improving radiologists' detection accuracy as well as the CAD performance.

In the detection of nodular shadows in chest radiographs, it is generally known that false-positive candidates are often produced near ribs, blood vessels, and their intersections [4]. If these components are deleted in preprocessing and the resulting image is used as the input image in CAD systems, it will be possible to delete the factors responsible for false-positive candidates before the main processing, which has previously been a problem.

Using segmented lung mask from previous subsection, rib suppression procedure is performed by oriented spatial Gabor filter. Before this procedure, we need an initial estimation of rib cage location. Nevertheless, rib cage segmentation is a challenging task in medical image processing and most of presented methods are based on a prior model. Since we have not such model and we want to do rib suppression automatically, we can suppress rib cage directly without detecting it.

After applying a preprocessing step, as mentioned in above (subsection 4- A), and enhancement of intensity and contrast, we use spatial Gabor filter based on vertical orientation to approximate ribs' location. As we already know spatial Gabor filter responds to spatial frequency components in predefined directions, and suppresses frequency components in other directions. Also we know that rib structure has high frequency component rather than other structures and background in chest radiography and extend horizontally. So based on prior medical knowledge about rib structure and the natural capabilities of spatial Gabor filter, we choose this filter for rib suppression in proposed method. Special frequency component in rib structure extraction is determined experimentally.

Consider the image of masked lung area in chest radiograph shown in Fig 5(a). If we apply a spatial Gabor filter oriented vertically on this image then it will give high responses wherever there are horizontal stripes present on the lung area as ribs. Fig 5(b) shows the amplitude of the response of such a vertically oriented spatial Gabor filter for the image. 

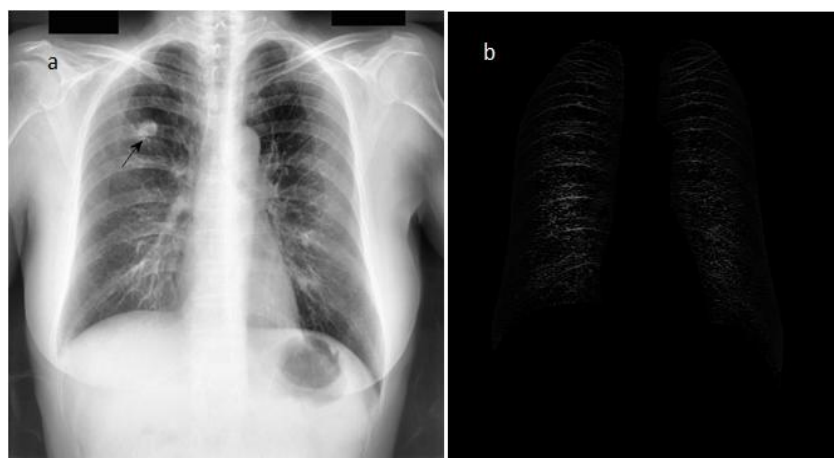

Figure 5: a) enhanced image b)result of applying spatial Gabor filter on enhanced image(a)( JPCLN006.jpg)

After model approximation of rib cage, and subtracting it from enhanced image, an image obtained with relatively obscured rib structure. On the other hand, applying this technique can cause some artifacts and noise; namely the signal-to-noise ratio (SNR) in the inter-rib regions of the subtracted images is reduced, which leads to a reduction in the diagnostic capability of the chest radiographs in these regions. Hence, there are some blood vessels distributed in lung area that increase false positive error in nodule detection system. In order to reduce blood vessel effects, noise and artifacts, one can use an adaptive Gaussian smoothing filter which enhance nodules because nodules naturally have Gaussian shapes. Fig. 6 shows images result before and after smoothing by Gaussian filter. In the images, nodule is indicated by an arrow.

\section{5- EXPERIMENTAL RESULTS}

A. Lung Segmentation:

The method was tested on 247 radiographs (154 of patients with lung nodules) in the standard JSRT database [3], which is the only known standard

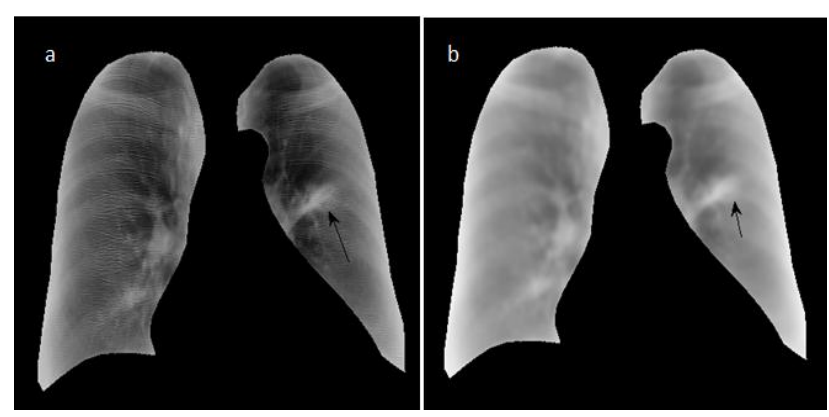

Figure 6: images obtained before (a) and after (b) smoothing by Gaussian filter by use of our technique. (JPCLN005.jpg) database publicly available. The images have been digitized with a $0.165 \mathrm{~mm}$ pixel size, a matrix size of $2048 \times 2048$, and 4096 gray levels.

Before processing, they have been down-sampled to a dimension of $512 * 512$ pixels; this experimental choice reduces the computational costs of the algorithm without affecting its performance.

To measure the performance of a segmentation algorithm, a 'goodness' index is required. For a two class segmentation problem, one can distinguish true positive (TP) area (correctly classified as object), false positive (FP) area (classified as object, but in fact background), false negative (FN) area (classified as background, but in fact object), and true negative (TN) area (correctly classified as background). From these values, measures such as accuracy, sensitivity, specificity and overlap can be computed given by the following equations.

$$
\begin{aligned}
& \text { Accuracy }=\frac{T P+T N}{T P+T N+F P+F N} \\
& \text { Sensitivity }=\frac{T P}{T P+F N} \\
& \text { Specificity }=\frac{T N}{T N+F P} \\
& \text { Overlap }=\frac{T P}{T P+F P+F N}
\end{aligned}
$$

Performance of the presented method in terms of abovementioned measures for JSRT database [3] is shown in Table 1.

Fig 7 shows the result of applying proposed method on some of images. As it can be seen in Fig.7, segmentation results show two areas: accurately segmented lung field (shown with red lines) and the area behind the heart and the diaphragm, where lung nodules may still are present (shown with blue lines).

Table1. Quantitative results for images in Fig.4

\begin{tabular}{|c|c|c|c|c|}
\hline & Accuracy & Sensitivity & Specificity & Overlay \\
\hline JPCLN003 & 0.9670 & 0.9835 & 0.9525 & 0.8632 \\
\hline JPCLN040 & 0.9660 & 0.9812 & 0.9575 & 0.9121 \\
\hline JPCLN100 & 0.9798 & 1.00 & 0.9726 & 0.9285 \\
\hline JPCLN152 & 0.9654 & 0.9889 & 0.9554 & 0.8948 \\
\hline $\begin{array}{c}\text { Average } \\
\text { result on } \\
\text { all images } \\
\text { of } \\
\text { database }\end{array}$ & 0.9625 & 0.9821 & 0.9565 & 0.9381 \\
\hline
\end{tabular}




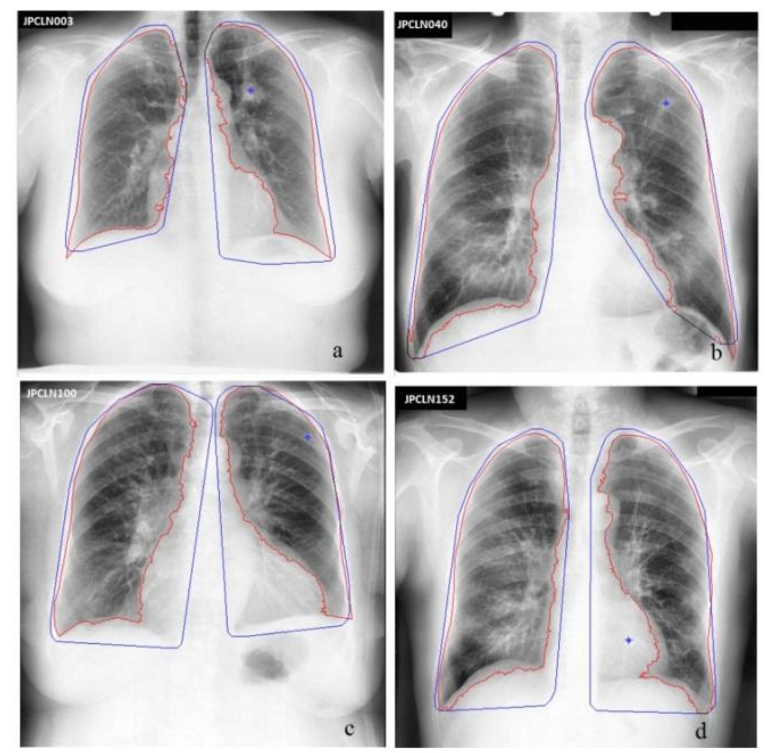

Figure7: Segmentation result of visible part (red line) and with nonvisible part (blue line) for images (a) JPCLN003 (b) JPCLN040 (c) JPCLN100 (d) JPCLN152.

B.VGinneken in [16] presented nine algorithms for the automatic delineation of lung fields in standard PA chest radiographs. The algorithms are based on different techniques: matching, pixel classification based on several combinations of features such as pixel intensity, location, entropy and etc., a rule-based scheme that finds lung contours using a general framework for the detection of oriented edges and ridges in images. Accuracy, sensitivity and specificity of all 9 systems on the complete test set are reported in table. 2 for comparison. PC stands for pixel classification. The number in parentheses denotes the system number. The systems are sorted according to accuracy.

Table 2. Result of all 9 systems on the complete test set[16]

\begin{tabular}{|l|c|c|c|}
\hline \multicolumn{1}{|c|}{ Method } & Accuracy & sensitivity & Specificity \\
\hline $\begin{array}{l}\text { Classification correction } \\
\text { (9) }\end{array}$ & 0.969 & 0.943 & 0.978 \\
\hline Rule-based (2) & 0.961 & 0.940 & 0.969 \\
\hline $\begin{array}{l}\text { PC int., entropy, corr. } \\
\text { location (8) }\end{array}$ & 0.956 & 0.912 & 0.972 \\
\hline $\begin{array}{l}\text { PC int., corrected } \\
\text { location (7) }\end{array}$ & 0.953 & 0.906 & 0.970 \\
\hline PC int. and location (6) & 0.933 & 0.854 & 0.966 \\
\hline Matching (1) & 0.907 & 0.766 & 0.946 \\
\hline PC location (4) & 0.898 & 0.784 & 0.947 \\
\hline PC int. (5) & 0.847 & 0.727 & 0.891 \\
\hline All negative (3) & 0.736 & 0 & 1 \\
\hline
\end{tabular}

They reported that their rule-based segmentation (system 2) required 4.1 seconds on average and their Pixel classification schemes required anywhere from 8 to 4 seconds. Their reclassification in system 9 requires the computation time of both the rule-based scheme, the pixel classifier of system 7, and reclassification. In total, that took 10.4 seconds on average. Segmentation by matching took about 24 seconds.

Average run time of our method with MATLAB 2010a in a Windows 7 platform with Core 2 Due 2.26 GHZ CPU and 4 GB of RAM is around 1 second for lung field segmentation of a chest radiograph. On the other hand, most the methods presented in the literature are indeed aimed at the definition of the most visible parts of the left and right lungs and i.e. the ones that are not hidden behind the diaphragm and the heart, while the non-visible area (which our method can distinguish) may contain nodules crucial for diagnosis.

\section{B. Improving the conspicuity of lung nodules by rib suppression:}

In this section, we quantify the effect of rib suppression on nodule conspicuity. The estimated softtissue images should reduce the rib shadows in radiographs and the contrast of soft-tissue in the lung (surrounded by lung parenchyma that predominantly contains air) should be better after filtering. We express the contrast of a nodule $\mathrm{C}_{\text {nod }}$ with its surroundings by the average intensity in the nodule minus the average intensity around the nodule. Images in the JSRT data set are annotated with the locations of nodules and their apparent diameters. The average intensity in the nodule $\left(\mathrm{g}_{\mathrm{N}}\right)$ is then calculated as the average intensity over the provided circle. For the intensity around the nodule $\left(g_{B}\right)$, the average intensity within a ring of twice the nodule diameter around the nodule is calculated.

$$
\mathrm{C}_{\text {nod }}=\mathrm{g}_{\mathrm{N}}-\mathrm{g}_{\mathrm{B}}
$$

To evaluate how much the contrast of a nodule is maintained, we defined a nodule contrast ratio (CR) represented by:

$$
\left.\mathrm{C}_{R}=\left(\mathrm{C}_{\text {nod }} \text { (original }\right)\right) /\left(\mathrm{C}_{\text {nod }}(\text { soft }- \text { tissue })\right)
$$

Average nodule contrast ratio over 154 nodules in all nodule-contained images of JSRT database was 1.1259 with a standard deviation of 0.0872 . The measures $C_{R}$ for all images are greater than one; the contrast of ribs is 
suppressed substantially in the soft-tissue image, whereas the contrast of a nodule is maintained. Thus, the visibility of the nodule is improved by suppression of the ribs around the nodule. Figure. 8 shows 2 cases with the best values among the 154 nodule cases, which is 1.4995 and 1.3476 that belong for images JPCLN006.jpg and JPCLN005.jpg of JSRT database, respectively.

Since most of studies in literature use dual energy subtraction method for suppression or use images obtained from this method in training phase, we cannot compare our result with them. Because there is no additional radiation exposure or specialized equipment required, it can also be applied to bedside portable [chest X-rays]. Additionally, our system does not require two acquisitions of the same patient, thereby eliminating distracting motion artifacts.

\section{6- CONCLUSION AND FUTURE WORK}

In this paper, a novel approach to extract the lung region in chest X-ray images is presented. Both qualitative and quantitative results show that we extract the lung region in X-ray image successfully. Experiment results demonstrate that the approach for lung region extraction is easy and fast in comparison with other

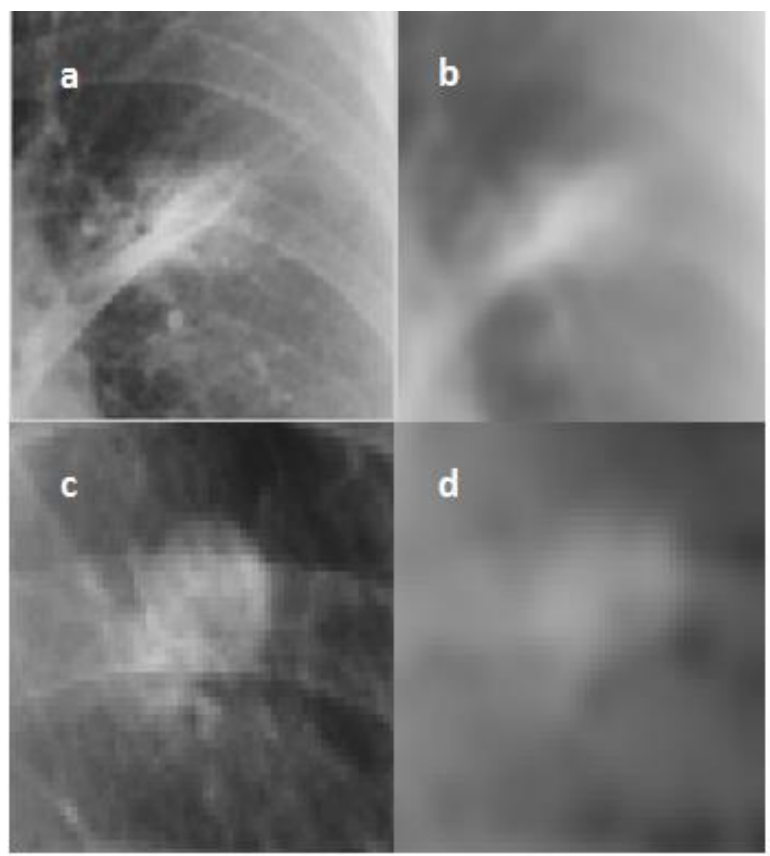

Figure 8: A close-up view of nodule region before $(a, c)$ and after(b,d) rib suppression in images JPCLN005.jpg (a,b), JPCLN006.jpg (c,d) methods. It can also be used in other medical images, such as CT, MRI, ultrasound images and so on. Also we proposed a new approach to filter obtained lung area by Gabor filer for suppressing rib cage. The main trend is clearly that the relative conspicuity of the nodules is increased in the inferred soft-tissue images compared to the original data. This indicates that the inferred softtissue images may be useful for nodule detection by either human observers or computer-aided diagnosis systems. The impact of this technique on the sensitivity of detection of lung nodules in digital chest radiographs will be investigated in a future observer performance study.

\section{7- ACKNOWLEDGMENT :}

We would like to thank Dr. Bram van Ginneken with Image Sciences Institute, the Netherlands, for kindly making available the manual segmentations of the JRST lung fields from his work in [20].

\section{8- References:}

[1] P. Campadelli and E. Casiraghi, "Lung Field Segmentation in Digital Postero-Anterior Chest Radiographs", ICAPR, LNCS 3687, pp. 1431-1440, 2005.

[2] B.van Ginneken, B.M.ter Haar Romeny, "Computer-aided diagnosis in chest radiography: a survey", Medical Imaging, IEEE Transactions on Medical Imaging, vol.20, no.12, pp. 1228-1241, Dec. 2001.

[3] P.K. Shah, J.H.M. Austin, C.S. White, P. Patel, L.B. Haramati, , G.D.N.Pearson, M.C. Shiau, Y.M. Berkmen "Missed nons mall cell lung cancer: radiographic findings of potentially resectable lesions evident only in retrospect" Radiology 226 (3), 235-241, 2003.

[4] M. Loog, B.van Ginneken, A. M. R.Schilham, , "Filter learning: Application to suppression of bony structures from chest radiographs," Medical Image Analysis, pp. 826-840, 2006.

[5] J. Shiraishi, S. Katsuragawa, J. Ikezoe, T. Matsumoto, T.Kobayashi, K. Komatsu, M. Matsui, H. Fujita, Y. Kodera, and K. Doi, Amer. J. Roentgenol. "Development of a digital image database for chest radiographs with and without a lung nodule: Receiver operating characteristic analysis of radiologists' detection of pulmonary nodules,", vol. 174, pp.71-74, 2000.

[6] M.Loog, B.van Ginneken, "Supervised segmentation by iterated contextual pixel classification", In: Proceedings of 16th International Conference on Pattern Recognition, pp. 925-928, 2002.

[7] L.Li, Y.Zheng, M.Kallergi, R.A.Clark, "Improved method for automatic identification of lung regions on chest radiographs", Academic Radiology , pp. 629-638, 2001.

[8] S.G.Armato, M.L.Giger, H.MacMahon, "Automated lung segmentation in digitized postero-anterior chest radiographs", Academic Radiology, pp. 245-255, 1998.

[9] X.W.Xu, K.Doi, "Image feature analysis for computer-aided diagnosis: detection of right and left hemidiaphragm edges and delineation of lung field in chest radiographs", Medical Physics 23(9), pp. 1613-1624, 1996.

[10] J.Duryea, J.M.Boone, "A fully automatic algorithm for the segmentation of lung fields in digital chest radiographic images", Medical Physics 22 (2), pp. 183-191, 1995. 
[11] E.Pietka, "Lung segmentation in digital chest radiographs.Journal of Digital Imaging”, pp. 79-84, 1994.

[12] M.S.Brown, L.S.Wilson, B.D.Doust, R.W.Gill, C.Sun, "Knowledge-based method for segmentation and analysis of lung boundaries in chest X-ray images", Computerized Medical Imaging and Graphics 22, pp. 463-477, 1998.

[13] M.F.McNitt-Gray, H.K.Huang, J.W.Sayre, "Feature selection in the pattern classification problem of digital chest radiograph segmentation", IEEE Transactions on Medical Imaging 14 (3), pp. 537-547, 1995.

[14] O.Tsujii, M.T.Freedman, S.K Mun, “Automated segmentation of anatomic regions in chest radiographs using an adaptivesized hybrid neural network". Medical Physics 25 (6), pp. 998 1007.

[15] N.F.Vittitoe, R.Vargas-Voracek, Jr.Floyd, "Identification of lung regions in chest radiographs using Markov Random Field modeling", Medical Physics 25 (6), pp. 976-985, 1998.

[16] B.van Ginneken, B.M.ter Haar Romeny, "Automatic segmentation of lung fields in chest radiographs", Medical Physics 27 (10), pp. 2445-2455, 2000.

[17] B.van Ginneken, Frangi, Staal, J.J., ter Haar Romeny, B.M.,Viergever, M.A, "Active shape model segmentation with optimal features", IEEE Transactions on Medical Imaging 21 (8), pp. 924-933, 2002.

[18] B. van Ginneken, S. Katsuragawa, ter Haar Romeny, B.M., Doi, K.,Viergever, M.A." Automatic detection of abnormalities in chest radiographs using local texture analysis". IEEE Transactions on Medical Imaging 21 (2), 139-149, 2002.

[19] C. Wang; Sh. Guo; X. Wu , "Segmentation of Lung Region for Chest X-Ray Images Based on Medical Registration And ASM," 3rd International Conference on Bioinformatics and Biomedical Engineering, p.p. 11-13, June 2009.

[20] B. Van Ginneken, Mikkel B. Stegmann, Marco Loog, " Segmentation of Anatomical Structures in Chest Radiographs using Supervised Methods: A Comparative Study on a Public Database", Medical Image Analysis, Vol. 10 No. 1 pp. 1940, 2006 .

[21] Suzuki, K.; Abe, H.; MacMahon, H.; Doi, K.; , "Imageprocessing technique for suppressing ribs in chest radiographs by means of massive training artificial neural network (MTANN)," Medical Imaging, IEEE Transactions on , vol.25, no.4, pp.406-416, April 2006

[22] W. R. Brody, D.M. Cassel, F. G. Sommer, L. A. Lehmann, A. Macovski,R. E. Alvarez, N. J. Pelc, S. J. Riederer, and A. L. Hall, "Dual-energy projection radiography: initial clinical experience," AJR. Am. J. Roentgenol., vol. 137, no. 2, pp. 201205, 1981.

[23] A. C. Bovik, M. Clark, and W. S. Geisler,"Multichannel texture analysis using localized spatial filters," IEEE Trans.vol. 12, no. 1, pp. 57-73, 1990.

[24] D. J. Gabor, "Theory of communication," IEEE Trans.vol. 93, no. 26, pp. 429-457, 1946.

[25] A. M. R. Schilham, B. v. Ginneken, and M. Loog, "A computeraided diagnosis system for detection of lung nodules in chest radiographs with an evaluation on a public database," science direct, pp. 247-258, 2006.

[26] N. Otsu , " A threshold selection method from gray-level histogram”, IEEE Trans SMC;SMC-9(1):62-6, 1979

\section{9- Bibliography}

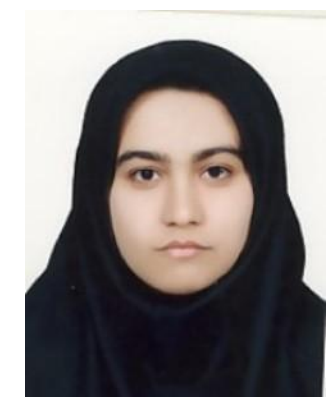

Elaheh Soleymanpour was born in Kashmar, Iran, in 1986. She received B.S. degree in computer engineering from Mazandaran University in 2008. Now she is M.S. Student in computer engineering in Ferdowsi University of Mashhad. Her research interests are medical image processing and computer vision.

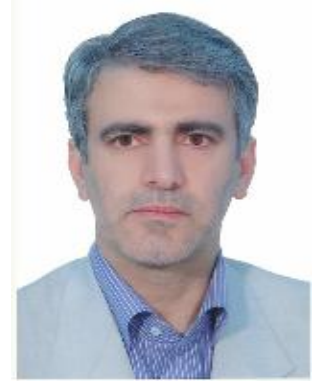

Hamid Reza Pourreza received B.S. degree in electrical engineering in Ferdowsi University of Mashhad in 1989. $\mathrm{He}$ received M.S degree in electrical engineering in Amirkabir University of Technology in 1993 and PHD degree in Amirkabir University of Technology in 2003. His areas of interests includes: computer vision, signal processing, hardware design and intelligent transportation systems. He has many publications in his area of interest.

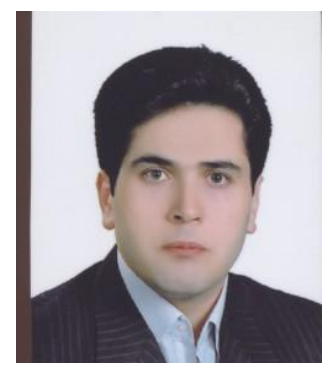

Emad Ansaripour was born in sabzevar, Iran, in 1976. He received MD degree in general physicion from mashhad medical science university in 2003 and he graduated from mashhad medical science university in Radiology major in 2011. Now he is doing his public commitments. His areas of interest includes: interventional radiology, musculoskeletal radiology, physics of diagnostic radiology

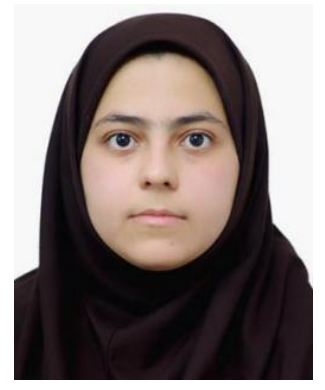

Mehri Sadoghi Yazdi was born in Kashmar, Iran, in 1983. She received B.S. degree in computer engineering from Azzahra University of Tehran in 2006 and then M.S. degree in Computer Engineering from Shahid Beheshti University of Tehran. Now she is PHD student in computer engineering in Ferdowsi University of Mashhad. Her research interests are image indexing and retrieval, fuzzy modeling and arithmetic. 